\title{
Novel design of smart sleep-lighting system for improving the sleep environment of children
}

\author{
Qun Wei ${ }^{\mathrm{a}}$ and, Jyung Hyun Lee ${ }^{\mathrm{b}}$ and Hee-Joon Park ${ }^{\mathrm{a}, *}$ \\ ${ }^{a}$ Department of Biomedical Engineering, School of Medicine, Keimyung University, Daegu, Korea \\ ${ }^{\mathrm{b}}$ Department of Biomedical Engineering, School of Medicine, Kyungpook National University, \\ Kyungpook National University Hospital, Daegu, Korea
}

\begin{abstract}
.
BACKGROUND: Children struggle to fall asleep by themselves because of their physiological characteristics. Therefore, research has been carried on various devices (such as a smartphone) to assist in improving the sleep quality of children. However, all such devices need to be controlled by parents and do not have functions for monitoring the sleep environment.

OBJECTIVE: In this paper, a smart sleep-lighting system that includes a sleep-lighting device and a smartphone dongle is developed to improve the sleep environment of children.

METHODS: The temperature, humidity, and luminance of the sleep environment are monitored and analyzed by the sleeplighting device to control multi-color light and audio components. The colored light emitted by the multi-color light can be adjusted to improve the sleep atmosphere. Also, the audio component can play white noise to induce sleep. In addition, parents can use a smartphone dongle with a multi-channel wireless communication method to monitor and control one or more lighting devices in different locations in real time.

RESULTS: For environmental monitoring, average difference between proposed device and commercial sensor from chamber setting temperature $15^{\circ} \mathrm{C}$ to $35^{\circ} \mathrm{C}$ was $0.588^{\circ} \mathrm{C} \pm 0.10^{\circ} \mathrm{C}$, and average error value of the humidity measurement was $0.74 \%$ at $40 \% \sim 60 \% \mathrm{RH}$. Also, the manufactured sleep-lighting device shows good performance in multi-color light emission, and playing of white noise. As result, the smartphone connected to the proposed smartphone dongle enables monitoring and control of the proposed lighting device in a wireless well.

CONCLUSIONS: The manufactured sleep-lighting device has a high-precision temperature and humidity sensor and a luminance sensor that can accurately monitor the sleeping environment. The lighting device can play white noise to induce sleep in children. Also, a multi-color LED light is operated via a smartphone application to improve the sleep atmosphere. The measured data will be sent to the lighting device and processed together with sleep environment data in order to improve the sleep quality. Additionally, the final system will be tested for real end-users with clinical experiments by sleep research center of a university hospital.
\end{abstract}

Keywords: Children, sleep environment, sleep aid, mood light, wireless multi-channel communication, smartphone dongle

\section{Introduction}

Sleep induction in children is a difficult issue, because children find it difficult to fall asleep by them-

\footnotetext{
${ }^{*}$ Corresponding author: Hee-Joon Park, Department of Biomedical Engineering, School of Medicine, Keimyung University, 1095, Dalgubeol-daero, Dalseo-Gu, Daegu, 42601, Korea. Tel.: +82 53580 3731; Fax: +82 53580 3731; E-mail: hjpark@kmu. ac.kr.
}

0928-7329/19/\$35.00 (C) 2019 - IOS Press and the authors. All rights reserved

This article is published online with Open Access and distributed under the terms of the Creative Commons Attribution NonCommercial License (CC BY-NC 4.0). 
selves for various physiological reasons [1-3]. Using white noise to induce children to sleep has been proposed, because it can assist with sleep induction. Spencer et al. studied two groups of 20 children between two and seven days old, $80 \%$ of whom fell asleep within 5 min in response to white noise, compared with only $25 \%$ of children in the control group [4]. Based on this, smartphone applications to induce sleep in children have been developed. Luxton et al., presented a study using smartphones to induce sleep with white noise technology. Lupton reported that many smartphone applications were developed for sleep induction [5-8]. However, the smartphone must be placed near the children until they are fully asleep. Any incoming telephone call will suspend the smartphone application operation and disturb the sleep induction. Also, the conditions of the sleep environment such as temperature, humidity, and brightness can affect the quality of children's sleep [9]. Thus, a current research has developed products with a Bluetooth communication for monitoring the sleep environment [10,11]. However, many families have more than one child; therefore, a multi-channel monitoring system is required.

Many studies have shown that different light colors can affect human moods. Red light is suitable for light in night, because it has the least power to shift circadian rhythm and suppress melatonin $[12,13]$. Also, colored lighting can modify the sleep atmosphere for children [14]. Many studies have demonstrated that from six months of age, children can see and distinguish colors as much as adults do [15-18].

Recently, several products for improving the sleeping environment have been proposed, such as LIFX, which is a Wi-Fi based, multi-color LED light bulb that can be controlled by smartphone. The device uses purple light to slow the heart rate and encourage relaxation, and deep red to aid sleep. However, the device only has a light function, and cannot monitor the sleep environment. Aura, made by Withings, can record temperature and luminosity. The music player function was designed to induce sleep. However, the device uses Bluetooth technology to connect with a smartphone, and is not suited to the family with more than one child.

In this paper, a smart sleep-lighting system for improving the sleep environment of children is presented. The proposed system is composed of a sleep-lighting device and a smartphone dongle. The lighting device has a single-chip relative humidity and temperature sensor, as well as an ultraviolet index, proximity, and ambient light sensor for sleep environment monitoring. The color and brightness of the sleep-lighting device can be adjusted to improve the sleep atmosphere based on the analyzed sleeping environment data $[19,20]$. Also, white noise can be played by an audio component in the sleep-lighting device to more efficiently induce sleep in children. A multi-channel RF wireless communication technology using a smartphone dongle was developed to simultaneously connect a smartphone and several lighting devices. Using an Android OS based smart phone application, users can monitor and control one or more lighting devices in different locations in real time. The concept of the proposed smart-lighting system for improving the sleep environment of children is shown in Fig. 1.

\section{Methods}

\subsection{Basic idea for proposed system}

Figure 2 shows a diagram of the proposed smart sleep-lighting system. The system is composed of two parts, a sleep-lighting device to monitor and improve the sleep environment, and a smartphone dongle to monitor and control the lighting device via wireless communication. In the sleep-lighting device, the humidity and temperature sensor, and ambient light sensor were controlled by an Acorn Reduced Instruction Set Computing Machine (ARM) based microprocessor. A high brightness red-green-blue 


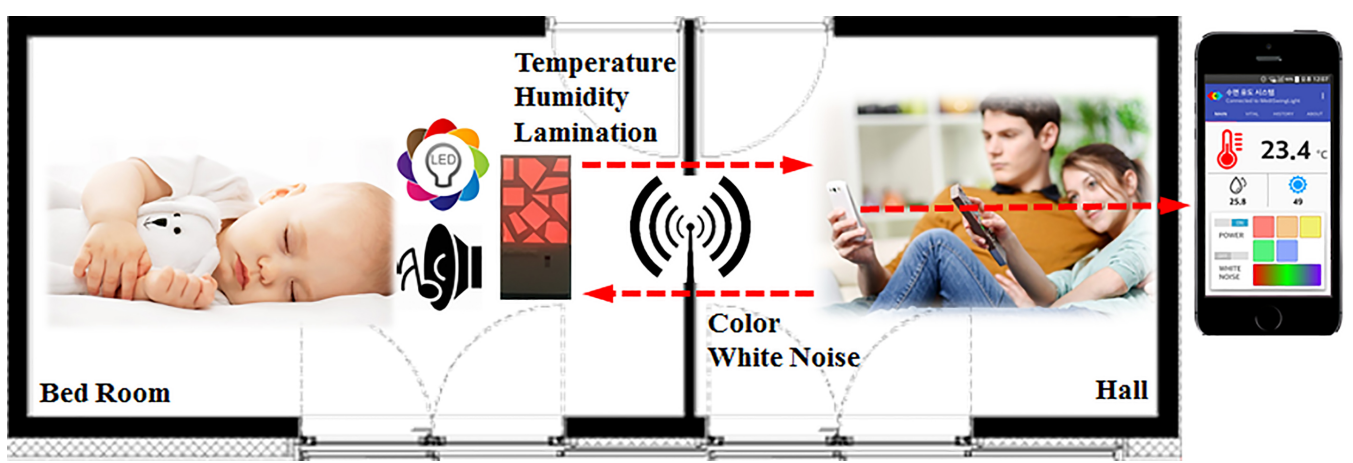

Fig. 1. Proposed smart-lighting system concept for improving the sleep environment of children.
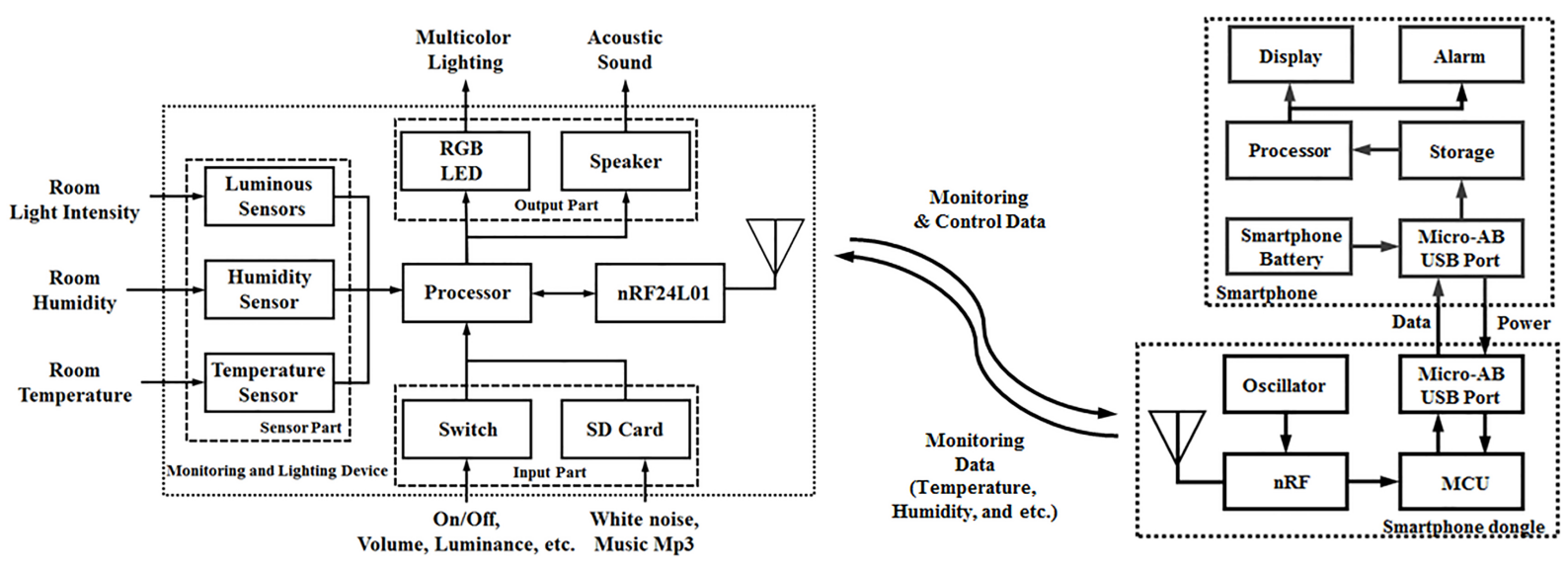

Fig. 2. Diagram of proposed smart sleep-lighting system.

light emitting diode (RGB LED) was used for emitting a variety of colored light. Also, a high resolution audio codec with a high quality speaker was included in the sleep-lighting device to play white noise which is stored in a micro-SD card as an mp3 file. The multi-color LED and audio component can be adjusted by the sleep-lighting device based on the analyzed sleep environment data or can be controlled by a smartphone application. For communication between the sleep-lighting device and the smartphone wireless condition, a low-power consumption multi-channel RF method was designed for the system. The smartphone dongle also has a low-power ARM processor for controlling the RF communication method and communicating with the smartphone. Using on-the-go (OTG) technology, the designed smartphone dongle can easily connect to the smartphone. Users can employ a developed smartphone application to monitor the sleeping environment and send instructions to control the sleep-lighting device.

\subsection{System design}

With respect to the system design, an EFM32GG series (Silicon Labs, USA) ARM processor was used as the main processor in the sleep-lighting device and smartphone dongle. The EFM32GG uses a CortexM3 CPU at speeds of up to $48 \mathrm{MHz}$. It is built on a low-power platform that uses low-energy techniques, fast wake-up times, and energy saving modes. In the sleep-lighting device, EFM32GG940F1024-QFN64 


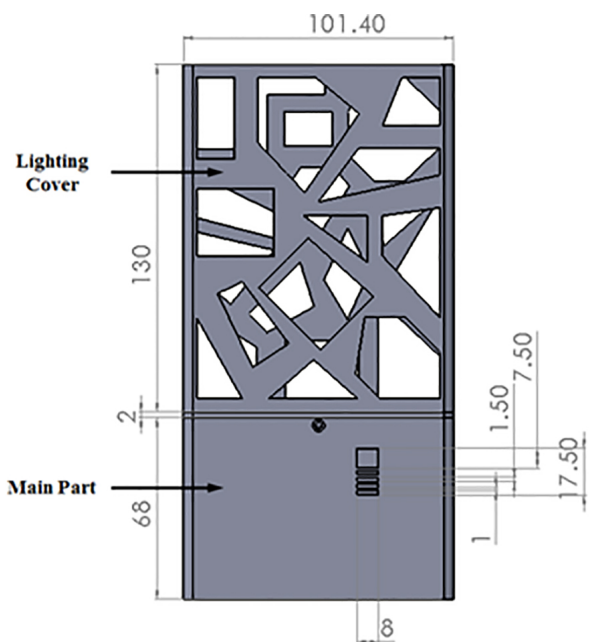

(a)

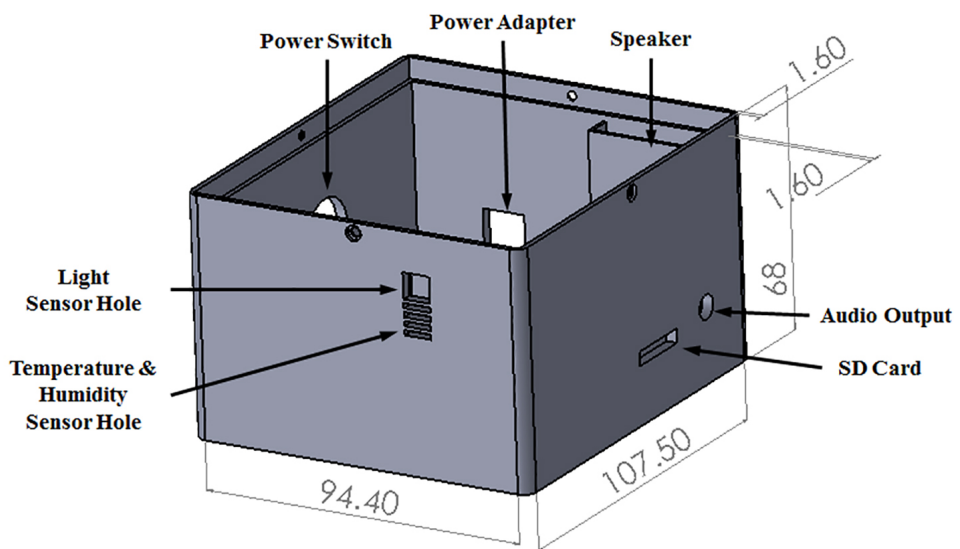

(b)

Fig. 3. Schematic of the 3D case model for the sleep-lighting device, (a) external view of 3D sleep-lighting device case model, (b) 3D model for the bottom part of the sleep-lighting device case.

is used as it includes 53 digital I/O pins that can connect with a variety of sensors. Additionally, $1024 \mathrm{kB}$ Flash and 128 RAM are used to assist with MP3 decoding. EFM32GG990F1024-BGA112 was included in the smart-phone dongle to control the RF transceiver and connect with the smartphone. This chip also has the same low power system and peripheral ports and is in a ball grid array (BGA) package, which minimizes the size of the printed circuit board (PCB) size. Also, the OTG function allows the microprocessor to connect with the smartphone via micro-USB. A relative humidity and temperature sensor Si7021 (Silicon Labs, USA) and a sensor monitoring ultraviolet index, gesture, proximity, and ambient light Si1145 (Silicon Labs, USA) were included in the design of the lighting device for monitoring the sleep environment. Si7021 has a measurement error of $\pm 3 \% \mathrm{RH}$ (maximum) and $\pm 0.4^{\circ} \mathrm{C}$ for humidity and temperature measurement. Also, Si1145 enables accurate light level monitoring under mixed lighting. The sensor can control the backlight automatically to improve the end user visual experience and reduce energy consumption. The two sensors have a wide operating voltage (1.9 to $3.6 \mathrm{~V}$ ), which is suitable for connecting with the EFM32 ARM processor.

A wireless transceiver nRF24L01 (Nordic Semiconductor, Nor-way) was used for wireless multichannel communication. The transceiver is a low power transceiver IC that operated on the $2.4 \mathrm{GHz}$ Industry-Science-Medical (ISM) band. Relay on a multi-communication technology; Multi-Ceiver, the receiver is able to simultaneously communicate with up to 128 transmitters. The wireless communication module was designed in the smartphone dongle connects to the smartphone via a micro-USB for communication with the lighting device.

Figure 3 shows a 3D case model of the proposed sleep-lighting device designed by SolidWorks (Dassault Systemes, USA), a solid modeling computer-aided design program. The case model is separated into two parts. One is designed as a box for fixing the system PCB, and the other is a frame for positioning the multi-colored lighting. The total size of the proposed light device is $104.4 \times 107.5 \times 200 \mathrm{~mm}$. The bottom of the sleep-lighting case is designed with several guide holes for fixing the electrical components such as the power switch of the main PCB as shown in Fig. 3b. To accurately measure the sleep environment, a temperature and humidity sensor hole is designed as a window-blind to protect the sensor. Additionally, on the temperature sensor hole, a square window is designed to allow the light 


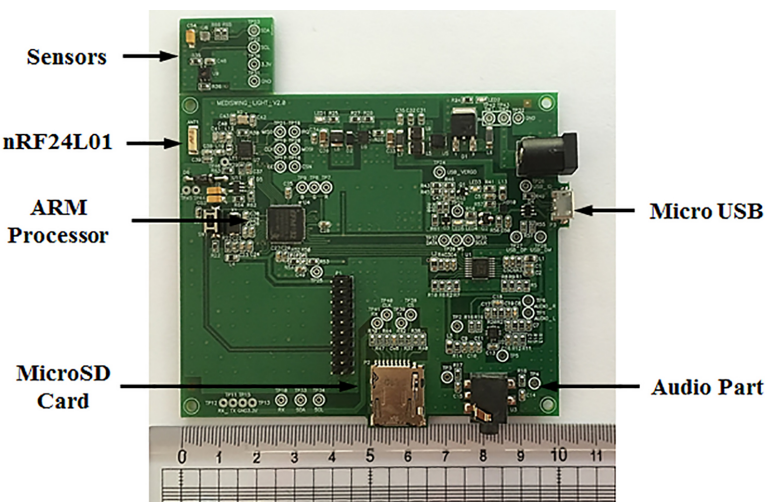

(a)

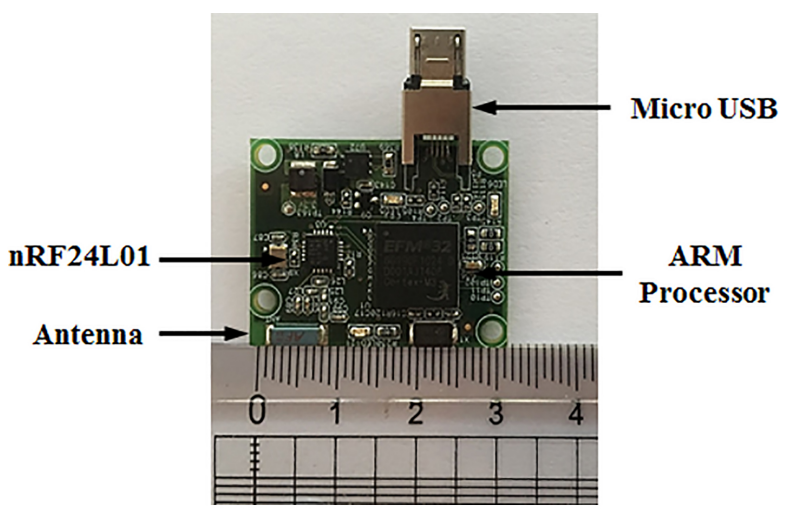

(b)

Fig. 4. Pictures of the manufactured smart sleep-lighting system PCBs, (a) Manufactured sleep-lighting device PCB, (b) Manufactured smartphone dongle PCB.

sensor to receive sufficient light. The audio jack hole is suitable for a 3.5 pi audio terminal for easy connection with external speakers. A RGB LED disk can be set up on the top of the bottom box to emit multi-colored light.

\section{Experiments}

\subsection{Proposed lighting device and smartphone dongle manufacturing}

Figure 4 illustrates the manufactured smart sleep-lighting system PCBs that include the sleep-lighting device and smartphone dongle. As shown in Fig. 4a, the lighting device was manufactured with a twolayer PCB and components with a 1608 size to fit the available space. The micro-SD card slot, microUSB port, audio output port, and power adapter were attached to the PCB. The temperature humidity sensor and lighting sensor were designed on a small PCB, which was connected to the main board. It is easy to separate from the main board, and fix the sensor hole of the sleep-lighting device case. The smartphone dongle PCB was manufactured with a four-layer PCB, as shown in Fig. 4b; all components were of SMD type with a size of 2012. This was done because the smartphone dongle must be as small as possible to connect to the smartphone. The micro-USB port on top of the PCB connects with the smartphone via a USB-OTG function. A $2.4 \mathrm{GHz}$ chip-shaped antenna was attached on the bottom of the PCB to reduce the electrical effect of the other components.

\subsection{Performance tests of the proposed lighting system}

The proposed sleep-lighting device was assembled with the designed case which was manufactured with a 3D printer from polylactic acid (PLA) material. The device was placed at the center of a temperature and humidity chamber (T2, YMRTC, Korea) to conduct temperature and humidity measurement performance tests, as shown in Fig. 5. Because of the optimal environment for sleeping is around $18^{\circ}$, with $50 \sim 60 \%$ for humidity [21]. The temperature and humidity in the chamber were respectively set as $15^{\circ} \mathrm{C}$ and $50 \%$ at an initial state in the temperature measurement performance test. The temperature was then elevated by $5^{\circ} \mathrm{C}$ every $30 \mathrm{~min}$, up to $35^{\circ} \mathrm{C}$. Because the temperature sensor for the chamber is 


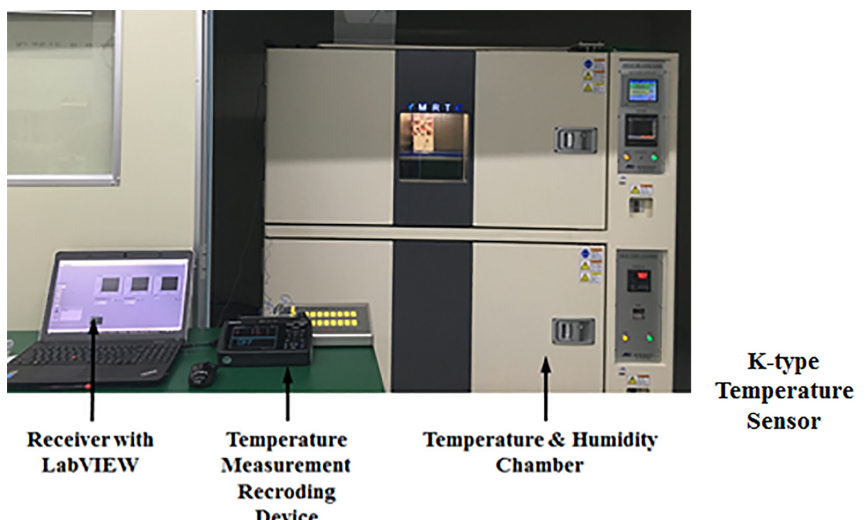

(a)

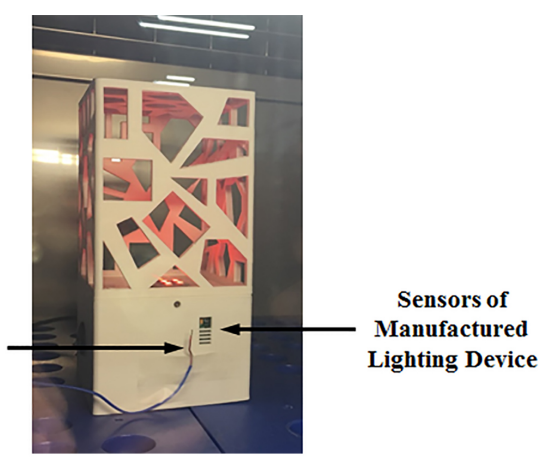

(b)

Fig. 5. Experiments for testing the temperature and humidity measurement performance of the proposed lighting device. (a) Image of the assembled sleep-lighting device used in the temperature and humidity measurement performance tests. (b) Using K-type thermocouple sensor to observe the temperature measurement performance of the sleep-lighting device.

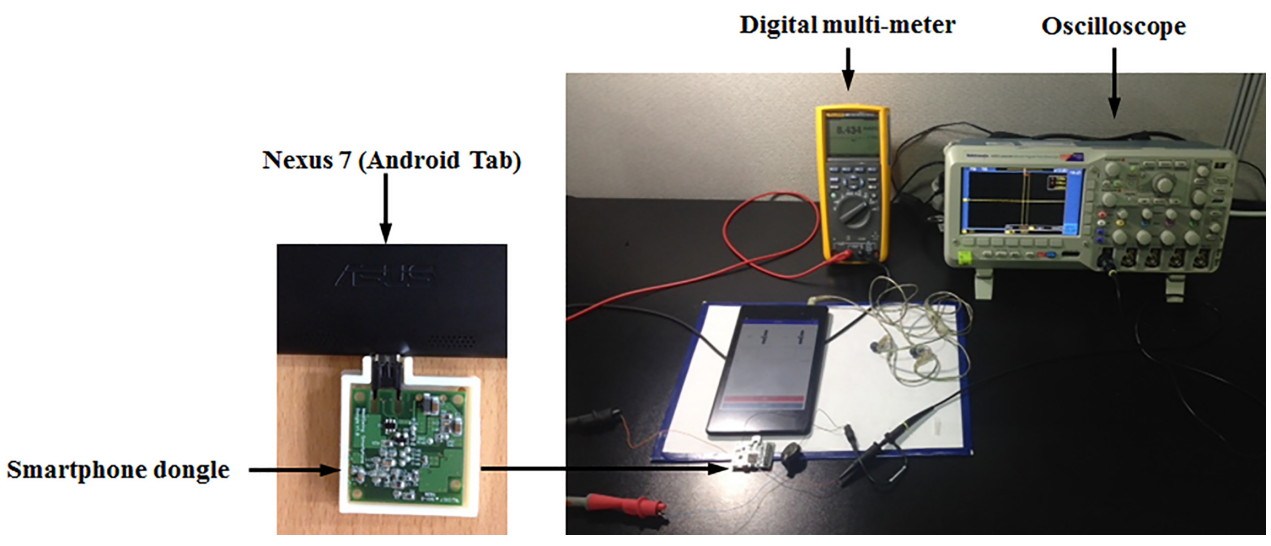

Fig. 6. Wireless communication performance testing of the manufactured smartphone dongle communicating with the sleeplighting device.

fixed on top of the chamber, the temperature value on the chamber display is not suitable for comparing the measured values of the proposed sleep-lighting device. A midi logger GL820 (GRAPHTEC, USA) was used to record the temperature of the inner chamber. As shown in Fig. 4b, a K-type temperature sensor was attached near the sensor hole of the proposed sleep-lighting device and connected with a midi logger for the changing temperature observation. For the humidity measurement performance test, the temperature was set at $25^{\circ} \mathrm{C}$ and the humidity was increased by $5 \%$ every $30 \mathrm{~min}$, in a range from $40 \%$ to $60 \%$. The temperature value transmitted to a PC that connected with a RF wireless communication test device. The received data was processed and simultaneously displayed using a developed LabVIEW program.

For testing the wireless communication performance of the designed smartphone dongle, the manufactured smartphone dongle was connected to a smart tab Nexus 7(ASUS, Taiwan) based on Android OS as shown in Fig. 6. The MISO port of the ARM processor was connected with an oscilloscope TBS1000 (Tektronix, USA) on the smartphone dongle to observe the data rate of the wireless communication. 


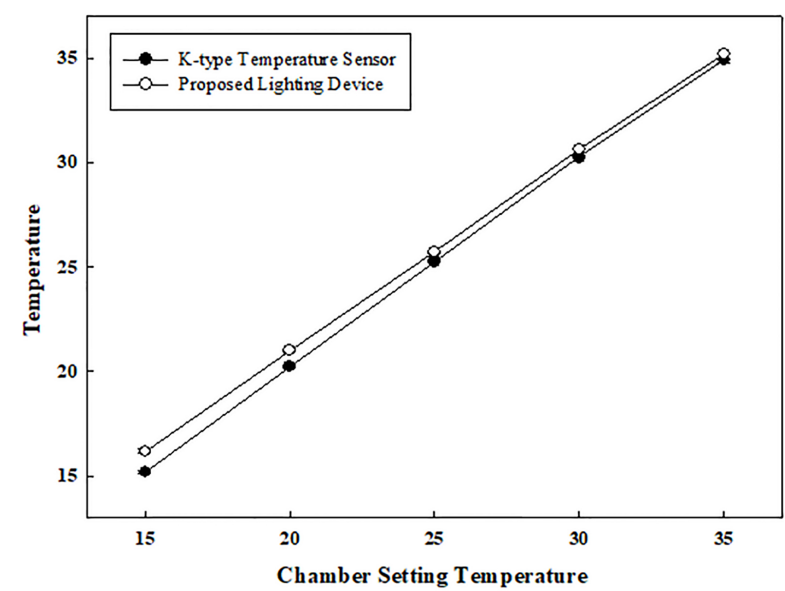

(a)

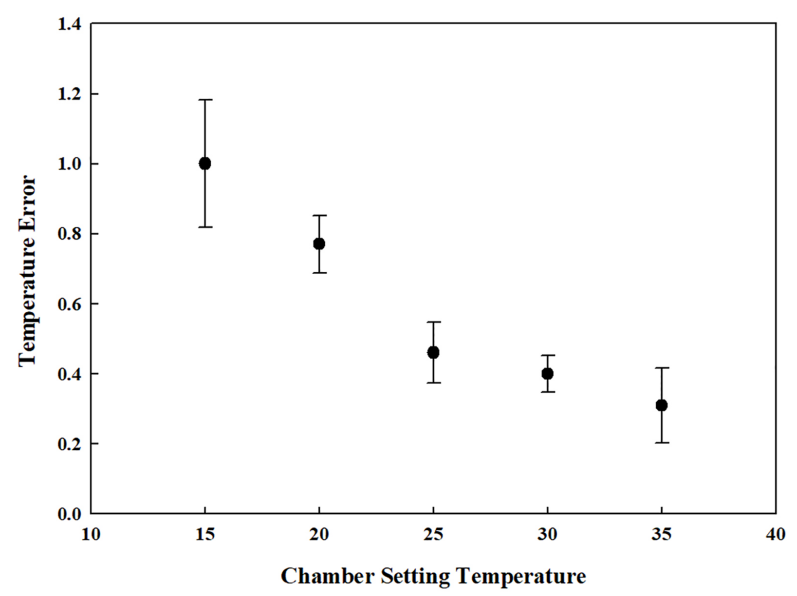

(b)

Fig. 7. Comparison of the proposed lighting device with a commercial temperature measurement device in terms of temperature measurement, (a) temperature measurement test between the proposed lighting device and the K-type temperature sensor, (b) error value of the measured temperature value between proposed device and the K-type temperature sensor.

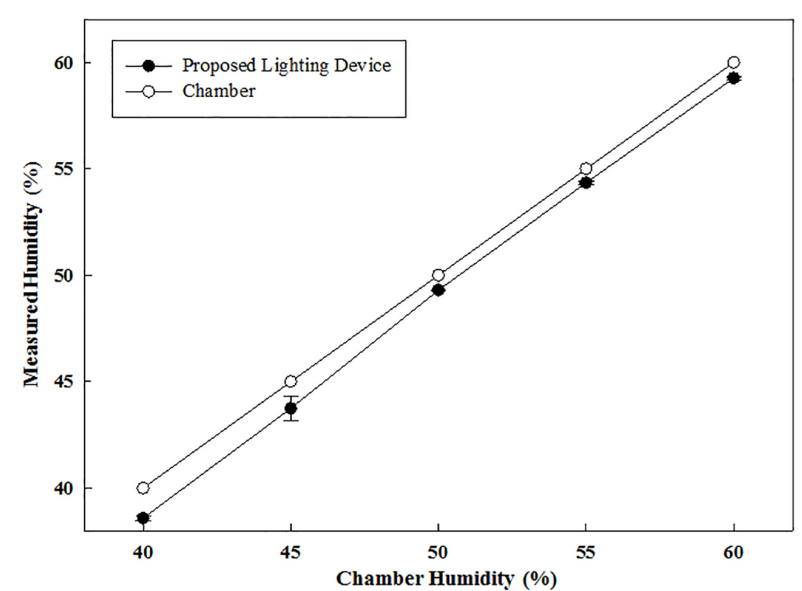

(a)

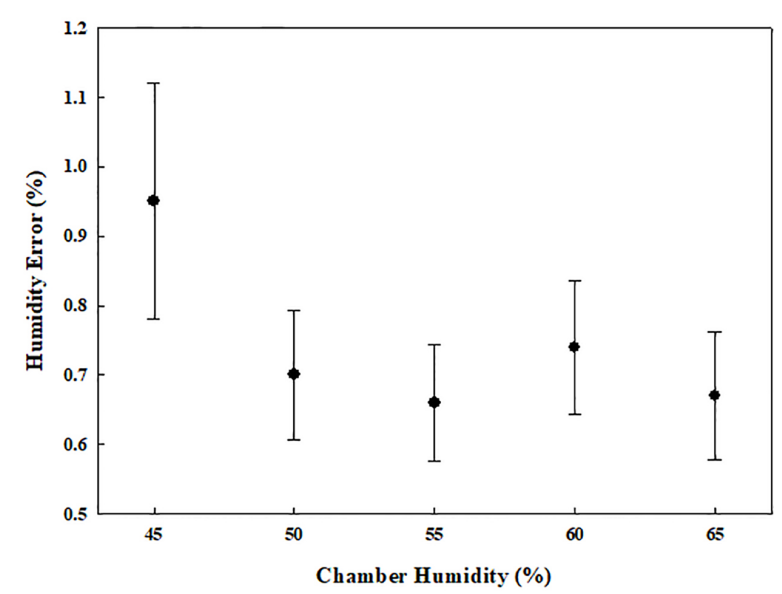

(b)

Fig. 8. Comparison of the proposed lighting device with humidity setting value of the chamber, (a) humidity measurement test between the proposed lighting device and the chamber displayed value, (b) error value of the measured humidity value between proposed device and the chamber displayed value.

\section{Results and discussion}

Experiment results of the proposed sleep-lighting device compared with the K-type temperature sensor in the performance tests of the temperature measurement are shown in Fig. 7. As illustrated in Fig. 7a, the temperature value measured by the sleep-lighting device was $1{ }^{\circ} \mathrm{C}$ higher than that measured by the k-type sensor at $15^{\circ} \mathrm{C}$. The difference in the two measured values decreased dramatically with temperature increased in the chamber. At $35^{\circ} \mathrm{C}$, the difference is only about $0.2^{\circ} \mathrm{C}$. All of the temperature value measured by the manufactured device was higher than the K-type temperature sensor due to the position of the designed sensor was close to the electrical elements, such as regulator; In addition, all of the elements were fixed in the shell of the lighting device that caused the heat dissipated slowly. Also, the 


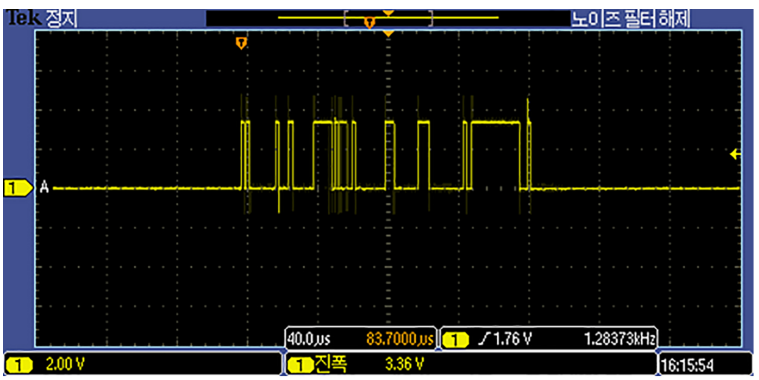

(a)

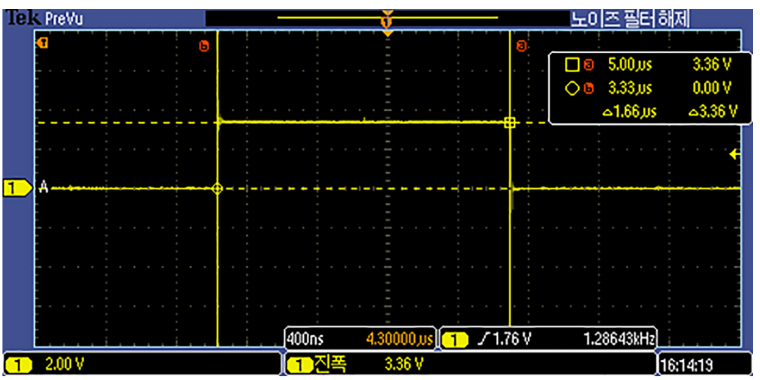

(b)

Fig. 9. Working performance of the smartphone dongle communicating wirelessly with the lighting device. (a) One data package is received from the lighting device, (b) data rate.

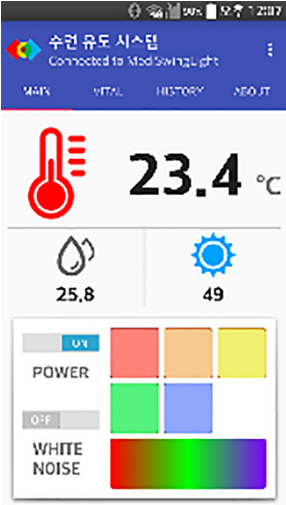

(a)
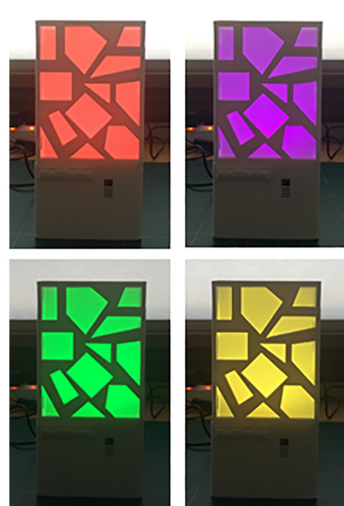

(b)

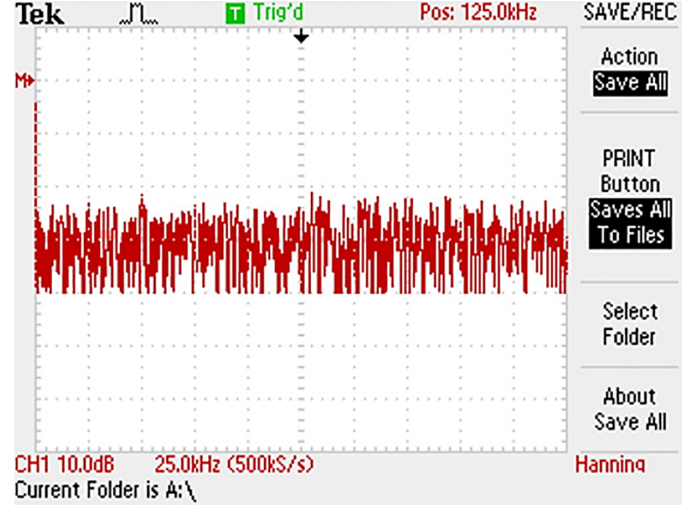

(c)

Fig. 10. Use of the smartphone application to control the manufactured lighting device, (a) developed smartphone application based on the Android operating system, (b) Using the smartphone application to control the manufactured sleep-lighting device. (c) White noise output of the lighting device in the frequency domain.

reason of the gap of the measurement values by two devices at the start point $\left(15^{\circ} \mathrm{C}\right)$ was larger than other temperature is assumed that the response of the mounted sensor in the manufactured device was slower than the referred K-type sensor about 5 seconds. Therefore, after through the sensor initialization, the temperature error reduced fast when the time increased. The correlation coefficients between temperature recorded by proposed device and commercial device were R-square equal to 0.9996 . The average difference between proposed device and K-type sensor from chamber setting temperature $15^{\circ} \mathrm{C}$ to $35^{\circ} \mathrm{C}$ was $0.588^{\circ} \mathrm{C} \pm 0.10^{\circ} \mathrm{C}$ as Fig. $7 \mathrm{~b}$ shown.

Figure 8 shows the experimental results of the lighting device in the humidity measuring performance test. As Fig. 8a shows, the measured humidity value was lower than that the displayed humidity value on the temperature and humidity chamber. As mentioned before, the sensor is close to the electrical elements and all of the elements were packaged in the designed shell, therefore, air around the sensor was drier than sensor of the chamber, and the moisture cannot be expelled quickly in the proposed device [22]. The error value of the proposed device and the chamber for humidity measurement test is shown in Fig. 8b. The largest error value was about $0.95 \%$ at start point $45 \%$ that also assumed to the maximum response time 17 seconds of the sensor without cover, and average error value was $0.74 \%$ at $40 \% \sim 60 \%$ chamber setting humidity that was lower than the typical error value $\pm 2 \%$ at $0 \sim 80 \% \mathrm{RH}$ which was mentioned on the datasheet. 
Figure 9 shows the working performance of the smartphone dongle when receiving data wirelessly from the lighting device. The lighting device transmits measured environmental data to the smartphone through the smartphone dongle. A single data package that includes measured temperature, humidity, and luminance values is shown in Fig. 9a, and the data rate is shown in Fig. 9b. The data transmission rate was about $650 \mathrm{kbps}$, and average power consumption was about $22 \mathrm{~mW}$ that was less than the ZigBee $30.69 \mathrm{~mW}$ and slightly higher than the Bluetooth $16.5 \mathrm{~mW}$ [23].

Figure 10 shows the developed smartphone application based on Android OS and the control of LED color changes. As shown in Fig. 10a, detailed the environmental information such as temperature, humidity and luminance are displayed on the application. Using the color indicator, the user can select the emitted light color to control the sleep-lighting device. The multi-colored sleep-lighting LED device emits a rainbow spectrum; the variety of colors in the developed smartphone application is shown in Fig. 10b. Such as the red color can help to improve the quality of sleep, and the blue color can help to increase the focusing attention that were presented by varied studies [24-27]. Figure 10c shows the output signal of the white noise function of the sleep-lighting device (used to induce sleep in children) which is processed by FFT to be determined in the frequency domain. The measured signal, which has a constant power spectral density, has the same characteristic as that of the white noise [28].

\section{Conclusion}

In this paper, a smart-lighting system was presented for improving the sleep environment of children. The system was composed of a sleep-lighting device and smartphone dongle. The manufactured sleep-lighting device has a high-precision temperature and humidity sensor and a luminance sensor that can accurately monitor the sleeping environment. The lighting device can play white noise to induce sleep in children. Also, a multi-color LED light is operated via a smartphone application to improve the sleep atmosphere [29]. The lighting device and smartphone communicate via an ISM band $2.4 \mathrm{GHz}$ low-power consumption RF method. Communication is mediated by a smartphone dongle connected to the smartphone by a micro-USB port. Therefore, a single smartphone can simultaneously connect to several lighting devices. The results showed that temperature and humidity can be accurately monitored using the proposed device, and that data can be transmitted to the smartphone in real time. Also, the multi-colored LED of the lighting device emits colored light selected using the smartphone application according to the analyzed sleep environment. In addition, the lighting de-vice can play high-quality white noise stored on a micro-SD card. The manufactured smartphone dongle was subjected to a wireless communication experiment and exhibited good performance with a $600 \mathrm{kbps}$ data rate and very low power consumption. In the future, a novel algorithm will be developed to improve the functionality of the proposed smart sleep-lighting system by simultaneously processing both the monitored bio-signals of children and data from the sleep environment. A health band for children is being developed to measure the bio-signals such as body temperature, electromyography (EMG) and heart rate. The measured data will be sent to the lighting device and processed together with sleep environment data in order to improve the sleep quality. Additionally, the final system will be tested for real end-users with clinical experiments by sleep research center of a university hospital.

\section{Acknowledgments}

This work was supported by the National Research Foundation of Korea (NRF) grant funded by the Korean government (MSIT) (NRF-2019R1A2C2004347). 


\title{
Conflict of interest
}

\author{
None to report.
}

\section{References}

[1] Stein MA, Mendelsohn J, Obermeyer WH, Amromin J, Benca R. Sleep and behavior problems in school-aged children. Pediatrics. 2001; 107(4): 60.

[2] Archbold KH, Pituch KJ, Panahi P, Chervin RD. Symptoms of sleep disturbances among children at two general pediatric clinics. The Journal of Pediatrics. 2002; 140(1): 97.

[3] Anders TF, Eiben LA. Pediatric sleep disorders: A review of the past 10 years. Journal of the American Academy of Child \& Adolescent Psychiatry. 1997; 36(1): 9.

[4] Spencer JA, Moran DJ, Lee A, Talbert D. White noise and sleep induction. Arch Dis Child. 1990; 1(65): 135.

[5] Luxton DD, McCann RA, Bush NE, Mishkind MC, Reger GM. mHealth for mental health: Integrating smartphone technology in behavioral healthcare. Professional Psychology: Research and Practice. 2011; 42(6): 505.

[6] Lupton D. Apps as Artefacts: Towards a Critical Perspective on Mobile Health and Medical Apps. Societies. 2014; 4(4): 602.

[7] Grifantini K. How's My sleep: Personal sleep trackers are gaining in popularity, but their accuracy is still open to debate. IEEE Pulse. 2014; 5(5): 14.

[8] Murnance EL, Huffaker D, Kossintes G. Mobile health apps: adoption, adherence, and abandonment, UbiComp/ ISWC'15; Adjunct; Osaka. 2015; p. 261.

[9] Imeri L, Opp MR. How (and why) the immune system makes us sleep. Nat Rev Neurosci. 2009; 10(3): 199.

[10] Othman MF, Shazail K. Wireless sensor network applications: A study in environment monitoring system. Procedia Engineering. 2012; 41: 1204.

[11] Choi JM, Choi BH, Seo JW, Sohn RH, Ryu MS, Yi W, Park KS. A system for ubiquitous health monitoring in the bedroom via a Bluetooth network and wireless LAN, $26^{\text {th }}$ Annual International Conference on IEEE; San Francisco: IEEE; 2004. p. 299.

[12] No SH. Emotional lighting system by feelux. Journal of Korean Society of Design Science. 2005; (1): 86.

[13] Blue light has a dark side, Exposure to blue light at night, emitted by electronics and energy-efficient lightbulbs, harmful to your health. Harv Health Lett. 2012; 37(7): 4.

[14] Kirov R, Kindelbur J, Banaschewski T, Rothenberger A. Sleep patterns in children with attention-deficit/hyperactivity disorder, tic disorder, and comorbidity. Journal of Child Psychology and Psychiatry. 2007; 48(6): 561.

[15] Bornstein MH, Qualities of color vision in infancy. Journal of Experimental Child Psychology. 1975; $19(3): 401$.

[16] Bornstein MH, Kessen W, Weiskopf S. Color vision and hue categorization in young human infants. Journal of Experimental Psychology: Human Perception and Performance. 1976; 2(1): 115.

[17] Adams RJ. An evaluation of color preference in early infancy. Infant Behavior and Development. 1987; $10(2): 143$.

[18] Brown AM. Development of visual sensitivity to light and color vision in human infants: A critical review. Vision Research. 1990; 30(8): 1159.

[19] Han YO, Kim DW. Sensitivity illumination system using biological signal. The Journal of the Korea Institute of Electronic Communication Sciences. 2014; 9(4): 499.

[20] Kim BS. The characteristics of the learning performance according to the indoor temperature of the Learning environment and the color of the learning materials. Journal of the Korea Academia-Industrial Cooperation Society. 2013; 14(2): 681.

[21] Huffington A. The Sleep Revolution: Transforming Your Life, One Night at a Time. Reprint ed. Harmony; 2017.

[22] Maluf KS, Morley RE Jr., Richter EJ, Klaesner JW, Mueller MJ. Monitoring in-shoe plantar pressures, temperature, and humidity: Reliability and validity of measures from a portable device. Archives of Physical Medicine and Rehabilitation. 2001; 82(8): 1119.

[23] Dementyev A, Hodges AS, Taylor S, Smith J. Power consumption analysis of Bluetooth low energy, ZigBee and ANT sensor nodes in a cyclic sleep scenario. IEEE International Wireless Symposium; Beijing: IEEE; 2013.

[24] Zhao J, Tian Y, Nie J, Xu J, Liu D. Red light and the sleep quality and endurance performance of Chinese female basketball players. Journal of Athletic Training. 2012; 47(6): 673.

[25] Jalil NA, Yunus RM, Said NS. Environmental colour impact upon human behavior: A review. Procedia - Social and Behavioral Sciences. 2012; 35: 54.

[26] Dalke H, Little J, Niemann E, Carmgoz N, Steadman G, Hill S, Stott L. Colour and lighting in hospital design. Optics \& Laser Technology. 2006; 38(4-6): 343. 
[27] Cahill J, Turner J, Barefoot H. Enhancing the student learning experience: The perspective of academic staff. Educational Research. 2010; 52(3): 283.

[28] Wu ZH, Huang NE. A study of the characteristics of White Noise using the empirical mode decomposition method. Proceedings of the Royal Society A. 2004; 460(2046): 1597.

[29] Chong WS, Hong CU, Kim NG. A study on human response to color light stimulation. Korean Journal of the Science of Emotion \& Sensibility. 2004; 7(4): 51. 